\title{
Pathology vs medical oncology: the crucial exchange of accurate information
}

\author{
S Dawood ${ }^{\star 1}$ \\ ${ }^{1}$ Department of Medical Oncology, Dubai Hospital, Department of Health and Medical Services, PO Box 8179, Dubai, UAE
}

Over the past two decades we have seen some truly exciting advances made in the biological understanding, diagnosis and management of breast cancer. We now recognise that breast cancer is a heterogeneous disease made of up of several subtypes. The introduction of an anthracycline polychemotherapy adjuvant regimen has reduced the 10 -year breast cancer mortality by approximately one-third and the introduction of the monoclonal antibody trastuzumab for the treatment of HER2-positive breast cancer has in the adjuvant setting been associated with an unprecedented relative improvement in disease-free survival by almost 50\%. Research is now focused on further improving prognostic outcome by exploring novel biomarkers that will better select patients requiring chemotherapy, identification and development of agents to overcome primary and secondary resistance of tumours to existing therapeutic agents as well as introducing new more efficacious chemotherapeutic and biological agents into adjuvant treatment protocols.

One potential prognostic marker that is currently being actively explored is the attainment of a pathological complete response (pCR) following a course of neoadjuvant chemotherapy. Pathological complete response over the past few years has garnered a lot attention raising several important questions. Can attaining a pCR improve prognostic outcome? Earlier retrospective studies indicated that attaining a pCR appeared to be associated with a significant improvement in prognostic outcome (Kuerer et al, 1999). Subsequent prospective clinical trials randomising patients between pre-operative and post-operative chemotherapy regimens were unable to show significant differences in prognostic outcome between the two strategies, however exploratory analysis indicated that a significant improvement in disease-free survival was observed among patients who did achieve a pCR compared with those who did not (Rastogi et al, 2008). Can we tailor treatment strategies based on whether a patient attains a pCR a not? Although there appears to a be a biological rational for the modification of chemotherapeutic regimens among patients with a poor initial response to chemotherapy clinical trials, exploring a change in chemotherapy in this cohort has not demonstrated clinically meaningful outcomes (Thomas et al, 2004; von
Minckwitz et al, 2008). Is there an ideal definition of pCR? A metanalysis of over 12000 patients enrolled in 12 neoadjuvant randomised controlled trials conducted by the FDA and presented at the recent San Antonio Breast Cancer Symposium concluded that the definition associated with the best prognostic outcome was one that included no invasive disease in the breast and axillary lymph nodes. The presence or absence of DCIS did not impact outcome (Cortazar et al, 2012). Are pCR rates different among different biological subtypes of breast cancer? In a pooled analysis of over 6000 patients enrolled in seven prospectively conducted neoadjuvant trials, rates of pCR were much higher among patients with more highly proliferative tumours, including the HER2enriched and triple-negative subtypes compared with hormone receptor-positive subtyes (von Minckwitz et al, 2012). Does the amount of residual disease following a course of neoadjuvant chemotherapy affect prognostic outcome? In a retrospective study of 382 patients treated with neoadjuvant anthracycline-based chemotherapy the group at the MD Anderson Cancer Center demonstrated that the presence of minimal residual disease was associated with a similar prognostic outcome as that associated with attaining a pCR with an inverse association observed between prognostic outcome and increasing burden of residual disease (Symmans et al, 2007).

Why the sudden fascination with pCR in the breast oncology community? This comes from the use of pCR for drug development and translational work but more importantly from the recent FDA proposal to use pCR as a surrogate end point as it is 'reasonably likely to predict clinical benefit', thus allowing for rapid drug efficacy assessment and accelerated FDA approval (Prowell and Pazdur, 2012). The Neo-tANGO trial is one of the several neoadjuvant trials looking at improving the current standard adjuvant regimen of anthracycline and taxanes. Investigators of this phase III trial that randomised 831 patients with early-stage breast cancer from 57 UK centres reported that the addition of neoadjuvant gemcitabine to an anthracycline/taxane regimen did not significantly impact pCR rates (Earl et al, 2009). In the present study (Provenzano et al, 2013), the investigators go on to conduct a central review of all pathology reports by both a 
pathologist and a medical oncologist looking at several pieces of reported data, including breast response, presence of axillary metastases, axillary node number and type of axillary surgery. The investigators asked essentially two important and interesting questions. First, was there variability in the quality and completeness of pathology reporting across the participating centres? The simple answer is yes, with the investigators observing a significant variation in reporting between laboratories. Among the 188 patients who achieved a pCR, $48 \%$ had no information regarding the number of specimen blocks taken to search for residual disease and in the remaining cases, a wide variability of blocks taken was noted ranging from 5 to 81 . Furthermore among the 637 patients who did not achieve a pCR, only $45 \%$ of reports had a comment on chemotherapy effect with a formal grading of chemotherapy response reported in less than $10 \%$ of cases. Of the reports reviewed, 36 patients had documented isolated tumour cells (ITC) in the axillary lymph nodes of which in 20 (55\%) cases these lymph nodes were reported as positive and in the remaining $16(45 \%)$ cases they were reported as negative lymph nodes. Although this did not impact the final primary end point of pCR, as all patients with ITC-positive lymph nodes had residual disease in the breast, the potential for impact on pCR definition is evident. Furthermore, one would assume that the variability and quality in reporting outside the confines of a clinical trial would be much higher and this may ultimately result in greater variability in PCR definition. Currently there are no published standard guidelines for the handling of specimens among patients who receive neoadjuvant chemotherapy. With pCR being increasingly used as a surrogate end point for prognostic outcome in clinical trials, it will become imperative to develop uniform methods for reporting response to chemotherapy to ensure reliability of this end point. Prognostic information is derived from both $\mathrm{pCR}$ and degree of residual disease burden. Although at present both variables do not affect clinical practice, they allow for the computation of risk of recurrence (e.g. using the residual cancer burden - RCB tool), an important piece of information that oncologists want to know and patients often ask for (Symmans et al, 2007; http://www. mdanderson.org/breastcancer_RCB). It is also likely that as data from future clinical trials become available, additional individualised treatment protocols may be implemented based on the degree of residual disease burden left behind.

Second, was there a discrepancy in interpreting pertinent information between the reviewing oncologist and pathologist? Among the 4130 data points collected, an $8.4 \%$ discrepancy rate between the two reviewers was observed. The most common discrepancy noted was in the interpretation of presence of chemotherapy response with the reviewing oncologist tending to consider any mention of fibrosis, necrosis or inflammation as presence of response to chemotherapy while the reviewing histopathologist required a specific statement about the presence or absence of chemotherapy response. Other points of disagreement noted included number of axillary lymph nodes, type of axillary surgery performed (SLNB vs ALND), classification of internal and intra mammary lymph nodes and interpretation of ITC. The discordance in interpretation highlights a need for more standard reporting procedures that are clear to the physician sitting in the clinic with the patient. Sources of discordance highlighted above may ultimately impact systemic and locoregional treatment practices as well as change eligibility of patients for clinical trials.

In summary, this study provides evidence for the important partnership between the oncologist, surgeon, radiation oncologist and pathologist required to ultimately provide the best individualised care for the patient suffering from breast cancer. A pathology report with too much information may be just as detrimental as one with too little, and with the increasing use of neoadjuvant treatment protocols both within and outside of clinical trials it is vital that standard guidelines be developed for handling of these breast specimens and essential components of information be clearly reported. As a medical oncologist involved in the care plan of a patient with breast cancer, I would personally like to see a single report with clearly mentioned data points needed for treatment decisions, including pre chemotherapy core biopsy results, formal grading of response to chemotherapy in the breast and axilla and type of axillary surgery done. There is also accumulating evidence of changes in hormone receptor status and HER2 status between core biopsies and residual disease post chemotherapy, which may in essence reflect tumour heterogeneity rather than a true change in receptor status (Hirata et al, 2009). Regardless although a change from positive receptor status to negative receptor status would not change treatment protocol, the reverse would alter management. As such, mention of receptor status on both the core biopsy and residual disease maybe helpful. The development of standard clear reporting procedures would ultimately reduce variability in reporting and misinterpretation, enhance accuracy and reliability of the pCR reporting in clinical trials and would allow for clear and accurate transfer of information to all physicians and the concerned patient.

\section{REFERENCES}

Cortazar P, Zhang L, Untch M, Mehta K, Costantino J, Wolmark N, Bonnefoi H, Cameron D, Gianni L, Valagussa P, Zujewski JA, Justice R, Loibl S, Wickerham L, Bogaerts J, Baselga J, Perou C, Blumenthal G, Blohmer J, Mamounas E, Bergh J, Semiglazov V, Prowell T, Eidtmann H, Paik S, Piccart M, Sridhara R, Fasching P, Swain SM, Slaets L, Tang S, Gerber B, Geyer C, Pazdur R, Ditsch N, Rastogi P, Eiermann W, von Mincwitz G (2012) Meta-analysis results from the Collaborative Trials in Neoadjuvant Breast Cancer (CTNeoBC). Cancer Res 72(Supp 24): 93 (abstract S1-S11).

Earl HM, Vallier A, Hiller L, Fenwick N, Iddawela M, Hughes-Davies L, Provenzano E, McAdam K, Hickish T, Caldas C (2009) Neo-tAnGo: A neoadjuvant randomized phase III trial of epirubicin/cyclophosphamide and paclitaxel \pm gemcitabine in the treatment of women with high-risk early breast cancer (EBC): First report of the primary endpoint, pathological complete response (pCR). J Clin Oncol 27: 15s, suppl; Abstract 522.

Hirata T, Shimizu C, Yonemori K, Hirakawa A, Kouno T, Tamura K, Ando M, Katsumata N, Fujiwara Y (2009) Change in the hormone receptor status following administration of neoadjuvant chemotherapy and its impact on the long-term outcome in patients with primary breast cancer. Br J Cancer 101(9): 1529-1536.

Kuerer HM, Newman LA, Smith TL, Ames FC, Hunt KK, Dhingra K, Theriault RL, Singh G, Binkley SM, Sneige N, Buchholz TA, Ross MI, McNeese MD, Buzdar AU, Hortobagyi GN, Singletary SE (1999) Clinical course of breast cancer patients with complete pathologic primary tumor and axillary lymph node response to doxorubicin-based neoadjuvant chemotherapy. J Clin Oncol 17(2): 460-469.

Provenzano E, Vallier A-L, Walland K, Champ R, Bowden S, Grier A, Fenwick N, Abraham J, Iddawela M, Caldas C, Hiller L, Dunn J, Earl HM (2013) A central review of histopathology reports after breast cancer neoadjuvant chemotherapy in the Neo-tAnGo Trial. Br J Cancer; e-pub ahead of print 8 January 2013; doi:10.1038/bjc.2012.547.

Prowell TM, Pazdur R (2012) Pathological complete response and accelerated drug approval in early breast cancer. N Engl J Med 366(26): 2438-2441.

Rastogi P, Anderson SJ, Bear HD, Geyer CE, Kahlenberg MS, Robidoux A, Margolese RG, Hoehn JL, Vogel VG, Dakhil SR, Tamkus D, King KM, Pajon ER, Wright MJ, Robert J, Paik S, Mamounas EP, Wolmark N (2008) Preoperative chemotherapy: updates of National Surgical Adjuvant Breast and Bowel Project Protocols B-18 and B-27. J Clin Oncol 26(5): 778-785.

Symmans WF, Peintinger F, Hatzis C, Rajan R, Kuerer H, Valero V, Assad L, Poniecka A, Hennessy B, Green M, Buzdar AU, Singletary SE, Hortobagyi GN, Pusztai L (2007) Measurement of residual breast cancer burden to predict survival after neoadjuvant chemotherapy. J Clin Oncol 25(28): 4414-4422.

Thomas E, Holmes FA, Smith TL, Buzdar AU, Frye DK, Fraschini G, Singletary SE, Theriault RL, McNeese MD, Ames F, Walters R, Hortobagyi GN (2004) The use of alternate, non-cross-resistant adjuvant 
chemotherapy on the basis of pathologic response to a neoadjuvant doxorubicin-based regimen in women with operable breast cancer: longterm results from a prospective randomized trial. J Clin Oncol 22(12): 2294-2302.

von Minckwitz G, Kümmel S, Vogel P, Hanusch C, Eidtmann H, Hilfrich J, Gerber B, Huober J, Costa SD, Jackisch C, Loibl S, Mehta K, Kaufmann M. German Breast Group (2008) Neoadjuvant vinorelbine-capecitabine versus docetaxel-doxorubicin-cyclophosphamide in early nonresponsive breast cancer: phase III randomized GeparTrio trial. J Natl Cancer Inst 100(8): 542-551.

von Minckwitz G, Untch M, Blohmer JU, Costa SD, Eidtmann H, Fasching PA, Gerber B, Eiermann W, Hilfrich J, Huober J, Jackisch C, Kaufmann M, Konecny GE, Denkert C, Nekljudova V, Mehta K, Loibl S (2012) Definition and impact of pathologic complete response on prognosis after neoadjuvant chemotherapy in various intrinsic breast cancer subtypes. J Clin Oncol 30(15): 1796-1804. 